\title{
Fuzzy Stochastic Differential Equations Driven by a Fuzzy Brownian Motion
}

\author{
Kumwimba S. Didier1, Walo O. Rebecca ${ }^{2}$, Mabela M. Rostin², Badibi O. Christopher1, \\ Kankolongo K. Patient ${ }^{1}$, Marcel Remon ${ }^{3}$
}

\author{
${ }^{1}$ Département de Mathématiques et Informatique, Faculté des Sciences, Université de Lubumbashi, Lubumbashi, République \\ Démocratique du Congo \\ ${ }^{2}$ Département de Mathématiques, Informatique et Statistiques, Faculté des Sciences et Technologies, Université de Kinshasa, \\ Kinshasa, République Démocratique du Congo \\ ${ }^{3}$ Département de Mathématiques, Faculté des Sciences, Université de Namur, Namur, Belgique \\ Email: didierkumwimba@gmail.com
}

How to cite this paper: Didier, K.S., Rebecca, W.O., Rostin, M.M., Christopher, B.O., Patient, K.K. and Remon, M. (2022) Fuzzy Stochastic Differential Equations Driven by a Fuzzy Brownian Motion. Journal of Applied Mathematics and Physics, 10, 641-655. https://doi.org/10.4236/jamp.2022.103046

Received: January 8, 2022

Accepted: March 5, 2022

Published: March 8, 2022

Copyright $\odot 2022$ by author(s) and Scientific Research Publishing Inc. This work is licensed under the Creative Commons Attribution International License (CC BY 4.0).

http://creativecommons.org/licenses/by/4.0/

\begin{abstract}
In our previous work, we study fuzzy Itô integrals driven by a fuzzy Brownian motion. In this article, we continue this study. The purpose of this paper is to study the weak uniqueness of fuzzy stochastic differential equations taking into account fuzzy Brownian motion. For instance, we construct the fuzzy stochastic differential equation driven by a fuzzy Brownian motion. To define and prove our results, we use the fuzzification, the alpha cut method and the Hausdorff distance between two fuzzy quantities. Some results are to our credit in this article like the instance, we construct the fuzzy stochastic differrential equation driven by fuzzy Brownian motion. Furthermore, we develop fuzzy Itô calculus driven by a fuzzy Brownian motion. Our result complement existing ones in that the fuzzy version of Brownian motion is taken into account.
\end{abstract}

\section{Keywords}

Adapted Fuzzy Stochastic Process, Fuzzy Itô Process, Fuzzy Itô Formula, Hukuhara Generalized Derivative

\section{Introduction}

In our previous research, we have dealt with Fuzzy Itô Integral Driven by a Fuzzy Brownian Motion and in the present work, we develop Fuzzy stochastic differential equations driven by a fuzzy Brownian motion [1].

Historically, the earliest approach for deterministic fuzzy differential equations 
was based on generalization of the Hukuhara derivative of a set-valued function. This was made by Puri and Ralescu in [2] and used by Kaleva in [3] [4].

Deterministic fuzzy stochastic differential equations have been developed due to investigations of dynamic systems that have several applications in the modeling of classical problems in control theory, physics, biology, engineering economics and finance. In this kind of study, random disturbances are the only source of uncertainty. To resolve these situations, stochastic analysis methods must be used. However, in most real problems, we face a second source of uncertainty: that of nature vague (imprecise, fuzzy, etc). In this paper, we will deal with fuzzy stochastic differential equations by taking into account the fuzzy Brownian motion. By concerning fuzzy stochastic differential equations containing a classical Brownian motion (not fuzzy), the following works can be viewed, Malinowski [5] [6] and [7]. Under certain conditions, we study the existence and uniqueness of solutions of fuzzy stochastic differential equations containing a fuzzy Brownian motion.

The novelty of the paper is the definition of the fuzzy Itô process driven by a fuzzy Brownian motion, the expansion and the generalization of the fuzzy itô formula. These are done in Section 3 and Section 4.

\section{Preliminaries and Notations}

This section presents some definitions and elementary concepts of fuzzy stochastic processes that will be used in the sequel. We assume that the reader is familiar with the fuzzy arithmetic, fuzzy functions and fuzzy random variables notions, for more details, see [8]-[13] and references therein.

Let us begin by the notion of the Hukuhara Generalized Derivative.

Definition 2.1. (Bede and Stefanini [14]) The Hukuhara generalized derivative of the fuzzy function $\tilde{f}:(a, b) \mapsto \mathbb{F}(\mathbb{R})$ at $x_{0} \in(a, b)$ is defined by

$$
\tilde{f}_{g H}^{\prime}\left(x_{0}\right)=\lim _{h \rightarrow 0} \frac{\tilde{f}\left(x_{0}+h\right) \ominus_{g H} \tilde{f}\left(x_{0}\right)}{h} .
$$

If $\tilde{f}_{g H}^{\prime}\left(x_{0}\right) \in \mathbb{F}(\mathbb{R})$ verifying (2.1) exists, we say that $\tilde{f}$ is Hukuhara generalized differentiable ( $\mathrm{gH}$-differentiable) at $x_{0}$.

Definition 2.2. (Bede and Stefanini $[14])$ Let $\tilde{f}:[a, b] \mapsto \mathbb{F}(\mathbb{R})$ and $x_{0} \in(a, b)$, with $\tilde{f}_{\alpha}^{L}(x)$ and $\tilde{f}_{\alpha}^{U}(x)$ all differentiable in $x_{0}$. We say that

1) $\tilde{f}$ is $[(i)-g H]$-differentiable in $x_{0}$ if

$$
\tilde{f}_{i . g H}^{\prime}\left(x_{0} ; \alpha\right)=\left[\left(\tilde{f}_{\alpha}^{L}\right)^{\prime}\left(x_{0}\right),\left(\tilde{f}_{\alpha}^{U}\right)^{\prime}\left(x_{0}\right)\right], \quad 0 \leq \alpha \leq 1,
$$

2) $\tilde{f}$ is $[(i i)-g H]$-differentiable in $x_{0}$ if

$$
\tilde{f}_{i i . g H}^{\prime}\left(x_{0} ; \alpha\right)=\left[\left(\tilde{f}_{\alpha}^{U}\right)^{\prime}\left(x_{0}\right),\left(\tilde{f}_{\alpha}^{L}\right)^{\prime}\left(x_{0}\right)\right], \quad 0 \leq \alpha \leq 1 .
$$

Definition 2.3 (Hinge point, Stefanini and Bede [14]) We say that the point $x_{0} \in(a, b)$ is a hindge point considering the differentiability of $\tilde{f}$, if in all neighbourhood $V$ of $x_{0}$, it exists $x_{1}<x_{0}<x_{2}$ such that 
Type (1) at the point $x_{1} \quad(2.2)$ is verified while (2.3) isn't and at the point $x_{2}$ (2.3) is satisfied while (2.2) isn't;

Type (2) at the point $x_{1}(2.3)$ is verified while (2.2) is not and at the point $x_{2} \quad(2.2)$ is satisfied while (2.3) isn't.

Definition 2.4. (Parametric representation of the Hukuhara partial derivative of order 1, Allahviranloo and al. [9]) Let $\tilde{f}(x, t): \mathbb{D} \mapsto \mathbb{F}(\mathbb{R}),\left(x_{0}, t_{0}\right) \in \mathbb{D}$ and $\tilde{f}_{\alpha}^{L}(x, t), \tilde{f}_{\alpha}^{U}(x, t)$ partially derivable functions with respect to $x$. We say that

1) $\tilde{f}(x, t)$ est $[(i)-p]$-differentiable with respect to $x$ at $\left(x_{0}, t_{0}\right)$ if

$$
\partial_{x_{i, g H}} \tilde{f}_{\alpha}\left(x_{0}, t_{0}\right)=\left[\partial_{x} \tilde{f}_{\alpha}^{L}\left(x_{0}, t_{0}\right), \partial_{x} \tilde{f}_{\alpha}^{U}\left(x_{0}, t_{0}\right)\right] .
$$

2) $\tilde{f}(x, t)$ is $[(i i)-p]$-differentiable with respect to $x$ at $\left(x_{0}, t_{0}\right)$ if

$$
\partial_{x_{i i . g H}} \tilde{f}_{\alpha}\left(x_{0}, t_{0}\right)=\left[\partial_{x} \tilde{f}_{\alpha}^{U}\left(x_{0}, t_{0}\right), \partial_{x} \tilde{f}_{\alpha}^{L}\left(x_{0}, t_{0}\right)\right] .
$$

\section{Definition 2.5. (Filtration and filtered probability space, H. Duminil-Copin} [15])

Let $T \in(0, \infty)$, we denote by $I:=[0, T]$ and let $(\Omega, \mathcal{F}, P)$ be a probability space. A family $\{\mathcal{F}\}_{t \in I}$ of $\sigma$-algebra on $\Omega$ is called a filtration if for all $t \geq s$, $\mathcal{F}_{s} \subset \mathcal{F}_{t} \subset \mathcal{F}$. A filtration is continuous if for all $t \geq 0, \mathcal{F}_{t}=\bigcap_{s \geq t} \mathcal{F}_{s}$.

$\left(\Omega,\{\mathcal{F}\}_{t \in I}, P\right)$ is called filtered probabilised space.

Definition 2.6. (Break Time, H. Duminil-Copin [15])

Let $(\Omega, \mathcal{F}, P)$ be a probability space and $\left\{\mathcal{F}_{t \geq 0}\right\}$ a filtration. A random variable $T$ with values in $\mathbb{R}^{+}$is called a break time if $\{T<t\}=\{\omega \in \Omega: T(\omega)<t\}$ is in $\mathcal{F}_{t}$ for all $t \geq 0$.

Definition 2.7. (Adapted fuzzy process, Malinowski [5])

A fuzzy stochastic process $\tilde{X}_{t}$ is $\left\{\mathcal{F}_{t}\right\}_{t \in I}$-adapted if for all $\alpha \in[0,1]$, the function $\left[\tilde{X}_{t}\right]_{\alpha}: \Omega \mapsto \mathbb{K}(\mathbb{R})$ is $\mathcal{F}_{t}$-mesurable for all $t \in I$. The fuzzy stochastic process $\tilde{X}_{t}$ is mesurable $\left[\tilde{X}_{t}\right]_{\alpha}: I \times \Omega \mapsto \mathbb{K}_{c}(\mathbb{R})$ is $\mathcal{B}(I) \otimes \mathcal{F}_{t}$-mesurable for all $\alpha \in[0,1]$, the function $\left[\tilde{X}_{t}\right]_{\alpha}$ is mesurable with respect to the $\sigma$-algebra $\mathcal{N}$ defined by

$$
\mathcal{N}:=\left\{A \in \mathcal{B}(I) \otimes \mathcal{F}_{t}: A^{t} \in \mathcal{F}_{t}, \forall t \in I\right\},
$$

Definition 2.8. Let $\tilde{X}_{t}$ be a fuzzy stochastic process. $\tilde{X}_{t}$ is $d_{\infty}$-continuous if almost all ( with respect to the probability measure $P$ ) his trajectories, that means $\tilde{X}(\cdot, \omega): I \mapsto \mathbb{F}_{c}(\mathbb{R})$ are $d_{\infty}$-continuous fuzzy functions.

$$
\tilde{\Phi}_{\alpha}^{U}(t, \omega)=\sum_{j \geq 0}\left(\tilde{E}_{j}(\omega)\right)_{\alpha}^{U} 1_{\left[j 2^{-n},(j+1)\right]}
$$

Definition 2.9. (Kumwimba and al. [1]) The simple fuzzy Itô integral $\tilde{\Phi}(t, \omega)$ driven by a Brownian motion is defined on $[S, T]$ by

$$
\int_{S}^{T} \tilde{\Phi}(t, \omega) \mathrm{d} \tilde{B}(t, \omega)=\bigoplus_{j \geq 0} \tilde{E}_{j}(\omega) \otimes\left[\tilde{B}\left(t_{j+1}, \omega\right) \ominus_{H} \tilde{B}\left(t_{j}, \omega\right)\right],
$$

where $t_{k}=t_{k}^{(n)}= \begin{cases}k 2^{-n} & \text { si } S \leq k 2^{-n} \leq T \\ S & \text { si } k 2^{-n}<S \\ T & \text { si } k 2^{-n}>T\end{cases}$ 
Remark 2.10. (Kumwimba and al. [1]) In the expression (2.8), $\tilde{B}\left(t_{j}\right)$ and $\tilde{E}_{j}$ are fuzzy numbers for all $j \geq 0$. Due to the stability of $\oplus, \otimes$ and $\ominus_{H}$ in $\mathcal{F}_{c}(\mathbb{R})$, The simple fuzzy Itô integral with respect to a fuzzy Brownian motion is a fuzzy number.

\section{Fuzzy Itô Process}

Let us define first the Fuzzy Itô process in the following manner.

Definition 3.1.

Let $\tilde{B}_{t}$ be a one-dimensional fuzzy Brownian motion defined upon a filtered $\left(\Omega, \mathcal{F},\{\mathcal{F}\}_{t \geq 0}, P\right)$.

We call fuzzy Itô process, a fuzzy process $\left\{\tilde{X}_{t}\right\}_{0 \leq t \leq T}$ with values in $\mathbb{F}_{c}(\mathbb{R})$ such that $\forall t \leq T$

$$
\tilde{X}_{t}=\tilde{X}_{0} \oplus \int_{0}^{t} \tilde{K}(s, \omega) \mathrm{d} s \oplus \int_{0}^{t} \tilde{H}(s, \omega) \mathrm{d} \tilde{B}_{s}, P \text {-a.e }
$$

with

1) $\tilde{X}_{0} \quad \mathcal{F}_{0}$-mesurable.

2) For all $\alpha \in(0,1], \quad i \in\{L, U\}$

$$
\begin{gathered}
P\left[\int_{0}^{t}\left|\tilde{K}_{\alpha}^{i}(s, \omega)\right| \mathrm{d} s<\infty \forall t \geq 0\right]=1, \\
\tilde{K} \in V_{F}(0, T) .
\end{gathered}
$$

3) For all $\alpha \in(0,1], \quad i \in\{L, U\}$

$$
P\left[\int_{0}^{t}\left|\tilde{H}_{\alpha}^{i}(s, \omega)\right|^{2} \mathrm{~d} s<\infty \forall t \geq 0\right]=1
$$

where $\tilde{H} \in V\left(0, T, \mathbb{F}_{c}(\mathbb{R})\right)$.

In the differential form, (3.1) is denoted by

$$
\mathrm{d} \tilde{X}_{t}=\tilde{K} \mathrm{~d} t \oplus \tilde{H} \mathrm{~d} \tilde{B}_{t} .
$$

Progressively, we can now present a one-dimensional fuzzy Itô process.

Now, let us give the fuzzy version of the integration by parts using generalised Hukuhara (Bede and Stefanini [14]).

Theorem 3.2.

Let $\tilde{F}(s, \omega)=\tilde{F}(s)$ be a continuous fuzzy function with bounded variation in $[0, t]$. Then

$$
\int_{0}^{t} \tilde{F}(s) \mathrm{d} \tilde{B}_{t}=\tilde{F}(t) \otimes \tilde{B}_{t} \ominus_{g H} \int_{0}^{t} \tilde{B}_{t} \otimes \tilde{F}_{g H}^{\prime}(s) \mathrm{d} s .
$$

Moreover, (3.3) is a fuzzy mumber.

Proof. We know that $\int_{0}^{t} \tilde{F}(s) \mathrm{d} \tilde{B}_{t}$ is a fuzzy number. Let $\alpha \in[0,1]$, we have $\tilde{F}_{\alpha}(s)=\left[\tilde{F}_{\alpha}^{L}(s), \tilde{F}_{\alpha}^{U}(s)\right]$ and $\tilde{F}_{\alpha}^{L}(s)$ and $\tilde{F}_{\alpha}^{U}(s)$ are differentiable-continuous. We thus obtain two cases (from the definition 2.2):

$$
\tilde{F}_{g H}^{\prime}(s, \alpha)=\left[\left(\tilde{F}_{\alpha}^{L}\right)^{\prime}(s),\left(\tilde{F}_{\alpha}^{U}\right)^{\prime}(s)\right]
$$

for $\tilde{F}[(i)-g H]$-differentiable and 


$$
\tilde{F}_{g H}^{\prime}(s, \alpha)=\left[\left(\tilde{F}_{\alpha}^{U}\right)^{\prime}(s),\left(\tilde{F}_{\alpha}^{L}\right)^{\prime}(s)\right],
$$

for $\tilde{F}[(i i)-g H]$-differentiable.

Suppose that $\tilde{F}$ est $[(i)-g H]$-differentiable.

Then, from the classical integration by parts, we obtain

$$
\int_{0}^{t} \tilde{F}_{\alpha}^{i}(s) \mathrm{d}\left(\tilde{B}_{t}\right)_{\alpha}^{j}=\tilde{F}_{\alpha}^{i}(t) \cdot\left(\tilde{B}_{t}\right)_{\alpha}^{j}-\int_{0}^{t}\left(\tilde{B}_{t}\right)_{\alpha}^{j} \cdot\left(\tilde{F}^{\prime}\right)_{\alpha}^{i}(s) \mathrm{d} s,
$$

for all $\alpha \in(0,1], i, j \in\{L, U\}$.

Due to the definition of Hukuhara difference and from this inequality $\mathrm{d}\left(\tilde{B}_{t}\right)_{\alpha}^{U}-\mathrm{d}\left(\tilde{B}_{t}\right)_{\alpha}^{U} \geq 0$.

$$
\begin{aligned}
& \text { This } \\
& {\left[\int_{0}^{t} \tilde{F}_{\alpha}^{L}(s) \mathrm{d}\left(\tilde{B}_{s}\right)_{\alpha}^{L}, \int_{0}^{t} \tilde{F}_{\alpha}^{U}(s) \mathrm{d}\left(\tilde{B}_{s}\right)_{\alpha}^{U}\right]} \\
& =\left[\tilde{F}_{\alpha}^{L}(t) \cdot\left(\tilde{B}_{t}\right)_{\alpha}^{L}-\int_{0}^{t}\left(\tilde{B}_{s}\right)_{\alpha}^{L} \cdot\left(\tilde{F}^{\prime}\right)_{\alpha}^{L}(s) \mathrm{d} s, \tilde{F}_{\alpha}^{U}(t) \cdot\left(\tilde{B}_{t}\right)_{\alpha}^{U}-\int_{0}^{t}\left(\tilde{B}_{s}\right)_{\alpha}^{U} \cdot\left(\tilde{F}^{\prime}\right)_{\alpha}^{U}(s) \mathrm{d} s\right] .
\end{aligned}
$$

By expressing the above integral in a sum of little integrals, for all $\alpha \in(0,1]$ we obtain

$$
\begin{aligned}
{\left[\int_{0}^{t} \tilde{F}(s) \mathrm{d} \tilde{B}_{t}\right]_{\alpha}=} & {\left[\int_{0}^{t} \tilde{F}_{\alpha}^{L}(s) \mathrm{d}\left(\tilde{B}_{s}\right)_{\alpha}^{L}, \int_{0}^{t} \tilde{F}_{\alpha}^{U}(s) \mathrm{d}\left(\tilde{B}_{s}\right)_{\alpha}^{U}\right] } \\
= & {\left[\tilde{F}_{\alpha}^{L}(t) \cdot\left(\tilde{B}_{t}\right)_{\alpha}^{L}, \tilde{F}_{\alpha}^{U}(t) \cdot\left(\tilde{B}_{t}\right)_{\alpha}^{U}\right] } \\
& \Theta_{g H}\left[\int_{0}^{t}\left(\tilde{B}_{s}\right)_{\alpha}^{L} \cdot\left(\tilde{F}^{\prime}\right)_{\alpha}^{L}(s) \mathrm{d} s, \int_{0}^{t}\left(\tilde{B}_{s}\right)_{\alpha}^{U} \cdot\left(\tilde{F}^{\prime}\right)_{\alpha}^{U}(s) \mathrm{d} s\right] \\
= & {\left[\tilde{F}_{\alpha}^{L}(t), \tilde{F}_{\alpha}^{U}(t)\right] \otimes_{i n t}\left[\left(\tilde{B}_{t}\right)_{\alpha}^{L},\left(\tilde{B}_{t}\right)_{\alpha}^{U}\right] } \\
& \Theta_{g H} \int_{0}^{t}\left[\left(\tilde{B}_{s}\right)_{\alpha}^{L},\left(\tilde{B}_{s}\right)_{\alpha}^{U}\right] \otimes_{i n t}\left[\left(\tilde{F}^{\prime}\right)_{\alpha}^{L}(s),\left(\tilde{F}^{\prime}\right)_{\alpha}^{U}(s)\right] \mathrm{d} s \\
= & {\left[\tilde{F}(t) \otimes \tilde{B}_{t}\right]_{\alpha} \Theta_{g H} \int_{0}^{t}\left[\tilde{B}_{t} \otimes \tilde{F}_{g H}^{\prime}(s)\right]_{\alpha} \mathrm{d} s } \\
= & {\left[\tilde{F}(t) \otimes \tilde{B}_{t} \Theta_{g H} \int_{0}^{t} \tilde{B}_{t} \otimes \tilde{F}_{g H}^{\prime}(s) \mathrm{d} s\right]_{\alpha} . }
\end{aligned}
$$

Thus the equality (3.3) is obtained from the equality of fuzzy mumbers.

\section{Fuzzy Itô Formula}

The most important result in the fuzzy stochastic integration is the fuzzy Itô formula. The Itô formula is also named variables change formula.

\subsection{Fuzzy Itô Formula Driven by a Fuzzy Brownian Motion}

\section{Theorem 4.1}

Let $\tilde{f}$ be a $\mathcal{C}^{2}$ class Hukuara differentiable fuzzy function in $\mathbb{F}_{c}(\mathbb{R})$ and let $\tilde{B}_{t}$ a fuzzy Brownian motion $\tilde{B}=\left\{\tilde{B}_{t}, t \geq 0\right\}$. So $\tilde{f}\left(\tilde{B}_{t}\right)$ is the fuzzy stochastic process defined by the formula

$$
\tilde{f}\left(\tilde{B}_{t}\right)=\tilde{f}\left(\tilde{B}_{0}\right) \oplus \int_{0}^{t} \tilde{f}_{g H}^{\prime}\left(\tilde{B}_{s}\right) \mathrm{d} \tilde{B}_{s} \oplus \frac{1}{2} \odot \int_{0}^{t} \tilde{f}_{g H}^{\prime \prime}\left(\tilde{B}_{s}\right) \mathrm{d} s .
$$

where $\left(\mathrm{d} \tilde{B}_{t}\right)^{2}=\mathrm{d} \tilde{B}_{t} \otimes \mathrm{d} \tilde{B}_{t}=\mathrm{d} s$. 
Proof. We only prove the cases where the fuzzy functions $\tilde{f}$ and $\tilde{f}_{g H}^{\prime}$ are either $[i-g H]$-differentiable or either $[i i-g H]$-differentiable and they do not contain the hinge points. Notice that the other cases can be treated similarly.

For the first case, the fuzzy functions $\tilde{f}\left(\tilde{B}_{t}\right), \tilde{f}_{g H}^{\prime}\left(\tilde{B}_{t}\right)$ and $\tilde{f}_{g H}^{\prime \prime}\left(\tilde{B}_{t}\right)$ have parametric representations for $\alpha \in[0,1]$;

$$
\begin{gathered}
{\left[\tilde{f}\left(\tilde{B}_{t}\right)\right]_{\alpha}=\left[\tilde{f}_{\alpha}^{L}\left(\tilde{B}_{\alpha}^{L}(t), \tilde{B}_{\alpha}^{U}(t)\right), \tilde{f}_{\alpha}^{U}\left(\tilde{B}_{\alpha}^{L}(t), \tilde{B}_{\alpha}^{U}(t)\right)\right],} \\
{\left[\tilde{f}_{g H}^{\prime}\left(\tilde{B}_{t}\right)\right]_{\alpha}=\left[\left(\tilde{f}_{\alpha}^{L}\right)^{\prime}\left(\tilde{B}_{\alpha}^{L}(t), \tilde{B}_{\alpha}^{U}(t)\right),\left(\tilde{f}_{\alpha}^{U}\right)^{\prime}\left(\tilde{B}_{\alpha}^{L}(t), \tilde{B}_{\alpha}^{U}(t)\right)\right]}
\end{gathered}
$$

and

$$
\left[\tilde{f}_{g H}^{\prime \prime}\left(\tilde{B}_{t}\right)\right]_{\alpha}=\left[\left(\tilde{f}_{\alpha}^{L}\right)^{\prime \prime}\left(\tilde{B}_{\alpha}^{L}(t), \tilde{B}_{\alpha}^{U}(t)\right),\left(\tilde{f}_{\alpha}^{U}\right)^{\prime \prime}\left(\tilde{B}_{\alpha}^{L}(t), \tilde{B}_{\alpha}^{U}(t)\right)\right] .
$$

The lower and the upper bounds of these $\alpha$-cuts are given by

$$
\begin{gathered}
\tilde{f}_{\alpha}^{L}\left(\tilde{B}_{\alpha}^{L}(t), \tilde{B}_{\alpha}^{U}(t)\right)=\min \left\{f\left(B_{t}\right): B_{t} \in \tilde{B}_{\alpha}(t)\right\}, \\
\tilde{f}_{\alpha}^{U}\left(\tilde{B}_{\alpha}^{L}(t), \tilde{B}_{\alpha}^{U}(t)\right)=\max \left\{f\left(B_{t}\right): B_{t} \in \tilde{B}_{\alpha}(t)\right\}, \\
\left(\tilde{f}_{\alpha}^{L}\right)^{\prime}\left(\tilde{B}_{\alpha}^{L}(t), \tilde{B}_{\alpha}^{U}(t)\right)=\min \left\{f^{\prime}\left(B_{t}\right): B_{t} \in \tilde{B}_{\alpha}(t)\right\}, \\
\left(\tilde{f}_{\alpha}^{U}\right)^{\prime}\left(\tilde{B}_{\alpha}^{L}(t), \tilde{B}_{\alpha}^{U}(t)\right)=\max \left\{f^{\prime}\left(B_{t}\right): B_{t} \in \tilde{B}_{\alpha}(t)\right\}, \\
\left(\tilde{f}_{\alpha}^{L}\right)^{\prime \prime}\left(\tilde{B}_{\alpha}^{L}(t), \tilde{B}_{\alpha}^{U}(t)\right)=\min \left\{f^{\prime \prime}\left(B_{t}\right): B_{t} \in \tilde{B}_{\alpha}(t)\right\}
\end{gathered}
$$

and

$$
\left(\tilde{f}_{\alpha}^{U}\right)^{\prime \prime}\left(\tilde{B}_{\alpha}^{L}(t), \tilde{B}_{\alpha}^{U}(t)\right)=\max \left\{f^{\prime \prime}\left(B_{t}\right): B_{t} \in \tilde{B}_{\alpha}(t)\right\} .
$$

In the same way, we could take $\left[\mathrm{d} \tilde{B}_{t}\right]_{\alpha}=\left[\mathrm{d} \tilde{B}_{\alpha}^{L}(t), \mathrm{d} \tilde{B}_{\alpha}^{U}(t)\right]$. From the classical Itô formula of the function $f\left(B_{t}\right)$ (Aggoun, Elliott [16]) we have

$$
f\left(B_{t}\right)=f\left(B_{0}\right)+\int_{0}^{t} f^{\prime}\left(B_{s}\right) \mathrm{d} B_{s}+\frac{1}{2} \int_{0}^{t} f^{\prime \prime}\left(B_{s}\right) \mathrm{d} s .
$$

Thus, the equations from (4.5) to (4.10) by taking the minimum and the maximum in (4.11) give for all $\alpha \in[0,1]$

$$
\begin{aligned}
{\left[\tilde{f}\left(\tilde{B}_{t}\right)\right]_{\alpha}^{L}=} & {\left[\tilde{f}\left(\tilde{B}_{0}\right)\right]_{\alpha}^{L}+\int_{0}^{t}\left(\tilde{f}_{\alpha}^{L}\right)^{\prime}\left(\tilde{B}_{\alpha}^{L}(t), \tilde{B}_{\alpha}^{U}(s)\right) \mathrm{d} \tilde{B}_{\alpha}^{L}(s) } \\
& +\frac{1}{2} \int_{0}^{t}\left(\tilde{f}_{\alpha}^{L}\right)^{\prime \prime}\left(\tilde{B}_{\alpha}^{L}(s), \tilde{B}_{\alpha}^{U}(s)\right) \mathrm{d} s
\end{aligned}
$$

and

$$
\begin{aligned}
{\left[\tilde{f}\left(\tilde{B}_{t}\right)\right]_{\alpha}^{U}=} & {\left[\tilde{f}\left(\tilde{B}_{0}\right)\right]_{\alpha}^{U}+\int_{0}^{t}\left(\tilde{f}_{\alpha}^{U}\right)^{\prime}\left(\tilde{B}_{\alpha}^{L}(t), \tilde{B}_{\alpha}^{U}(s)\right) \mathrm{d} \tilde{B}_{\alpha}^{U}(s) } \\
& +\frac{1}{2} \int_{0}^{t}\left(\tilde{f}_{\alpha}^{U}\right)^{\prime \prime}\left(\tilde{B}_{\alpha}^{L}(s), \tilde{B}_{\alpha}^{U}(s)\right) \mathrm{d} s .
\end{aligned}
$$

Hence, for all $\alpha \in[0,1]$, 


$$
\begin{aligned}
{[} & \left.\tilde{f}\left(\tilde{B}_{t}\right)\right]_{\alpha} \\
= & {\left[\left[\tilde{f}\left(\tilde{B}_{0}\right)\right]_{\alpha}^{L}+\int_{0}^{t}\left(\tilde{f}_{\alpha}^{L}\right)^{\prime}\left(\tilde{B}_{\alpha}^{L}(s), \tilde{B}_{\alpha}^{U}(s)\right) \mathrm{d} \tilde{B}_{\alpha}^{L}(s)+\frac{1}{2} \int_{0}^{t}\left(\tilde{f}_{\alpha}^{L}\right)^{\prime \prime}\left(\tilde{B}_{\alpha}^{L}(s), \tilde{B}_{\alpha}^{U}(s)\right) \mathrm{d} s,\right.} \\
& {\left.\left[\tilde{f}\left(\tilde{B}_{0}\right)\right]_{\alpha}^{U}+\int_{0}^{t}\left(\tilde{f}_{\alpha}^{U}\right)^{\prime}\left(\tilde{B}_{\alpha}^{L}(s), \tilde{B}_{\alpha}^{U}(s)\right) \mathrm{d} \tilde{B}_{\alpha}^{U}(s)+\frac{1}{2} \int_{0}^{t}\left(\tilde{f}_{\alpha}^{U}\right)^{\prime \prime}\left(\tilde{B}_{\alpha}^{L}(s), \tilde{B}_{\alpha}^{U}(s)\right) \mathrm{d} s\right] } \\
= & {\left[\left[\tilde{f}\left(\tilde{B}_{0}\right)\right]_{\alpha}^{L},\left[\tilde{f}\left(\tilde{B}_{0}\right)\right]_{\alpha}^{U}\right] } \\
& \oplus_{\text {int }}\left[\int_{0}^{t}\left(\tilde{f}_{\alpha}^{L}\right)^{\prime}\left(\tilde{B}_{\alpha}^{L}(s), \tilde{B}_{\alpha}^{U}(s)\right) \mathrm{d} \tilde{B}_{\alpha}^{L}(s), \int_{0}^{t}\left(\tilde{f}_{\alpha}^{U}\right)^{\prime}\left(\tilde{B}_{\alpha}^{L}(s), \tilde{B}_{\alpha}^{U}(s)\right) \mathrm{d} \tilde{B}_{\alpha}^{U}(s)\right] \\
& \oplus_{\text {int }}\left[\frac{1}{2} \int_{0}^{t}\left(\tilde{f}_{\alpha}^{L}\right)^{\prime \prime}\left(\tilde{B}_{\alpha}^{L}(s), \tilde{B}_{\alpha}^{U}(s)\right) \mathrm{d} s, \frac{1}{2} \int_{0}^{t}\left(\tilde{f}_{\alpha}^{U}\right)^{\prime \prime}\left(\tilde{B}_{\alpha}^{L}(s), \tilde{B}_{\alpha}^{U}(s)\right) \mathrm{d} s\right] \\
= & {\left[\tilde{f}\left(\tilde{B}_{0}\right)\right]_{\alpha} \oplus_{\text {int }}\left[\int_{0}^{t} \tilde{f}_{g H}^{\prime}(\tilde{B}(s)) \mathrm{d} \tilde{B}(s)\right]_{\alpha} \oplus_{\text {int }} \frac{1}{2} \odot_{i n t}\left[\int_{0}^{t} \tilde{f}_{g H}^{\prime \prime}(\tilde{B}(s)) \mathrm{d} s\right] . }
\end{aligned}
$$

Hence, for every $\alpha \in[0,1]$,

$$
\left[\tilde{f}\left(\tilde{B}_{t}\right)\right]_{\alpha}=\left[\tilde{f}\left(\tilde{B}_{0}\right) \oplus \int_{0}^{t} \tilde{f}_{g H}^{\prime}\left(\tilde{B}_{s}\right) \mathrm{d} \tilde{B}_{s} \oplus \frac{1}{2} \odot \int_{0}^{t} \tilde{f}_{g H}^{\prime \prime}\left(\tilde{B}_{s}\right) \mathrm{d} s\right]_{\alpha} .
$$

which establishes by definition, the equality sought.

\subsection{Fuzzy Itô Formula of a Fuzzy Itô Process}

Let us start this part by the following theorem.

\section{Theorem 4.2.}

Let $\tilde{X}_{t}$ Fuzzy d'Itô process such that

$$
\mathrm{d} \tilde{X}_{t}=\tilde{K} \mathrm{~d} t \oplus \tilde{H} \mathrm{~d} \tilde{B}_{t} .
$$

Let $\tilde{G}(t, x) \in \mathcal{C}\left([0, \infty) \times \mathbb{F}_{c}(\mathbb{R})\right)$. Then

$$
\tilde{Y}_{t}=\tilde{G}\left(t, \tilde{X}_{t}\right)
$$

is also a fuzzy Itô process and

$$
\mathrm{d} \tilde{Y}_{t}=\partial_{t_{g H}} \tilde{G}\left(t, \tilde{X}_{t}\right) \mathrm{d} t \oplus \partial_{x_{g H}} \tilde{G}\left(t, \tilde{X}_{t}\right) \otimes \mathrm{d} \tilde{X}_{t} \oplus \frac{1}{2} \odot \partial_{x x_{g H}} \tilde{G}\left(t, \tilde{X}_{t}\right) \otimes\left(\mathrm{d} \tilde{X}_{t}\right)^{2}
$$

where $\left(\mathrm{d} \tilde{X}_{t}\right)^{2}=\mathrm{d} \tilde{X}_{t} \otimes \mathrm{d} \tilde{X}_{t}$ is computed according

$$
\mathrm{d} t \cdot \mathrm{d} t=0, \mathrm{~d} t \odot \mathrm{d} \tilde{B}_{t}=\mathrm{d} \tilde{B}_{t} \odot \mathrm{d} t=\tilde{0}, \mathrm{~d} \tilde{B}_{t} \otimes \mathrm{d} \tilde{B}_{t}=1_{\{\mathrm{d} t\}}=\mathrm{d} t .
$$

Proof. Let $K(s, \omega)$ and $H(s, \omega)$ in $\mathbb{F}_{c}(\mathbb{R})$, such that

$$
\mathrm{d} \tilde{X}_{t}=\tilde{K} \mathrm{~d} t \oplus \tilde{H} \mathrm{~d} \tilde{B}_{t} .
$$

Then we have for all $\alpha \in[0,1]$, two cases according that

$$
\left[\mathrm{d} \tilde{X}_{t}\right]_{\alpha}=\left[\mathrm{d}\left(\tilde{X}_{t}\right)_{\alpha}^{L}, \mathrm{~d}\left(\tilde{X}_{t}\right)_{\alpha}^{U}\right]=\left[\tilde{K} \mathrm{~d} t \oplus \tilde{H} \mathrm{~d} \tilde{B}_{t}\right]_{\alpha}=\left[L_{1}, L_{2}\right],
$$

with $L_{1}=\min _{i \in J} l_{i}$ and $L_{2}=\max _{i \in J} l_{i}$ such that $J=\{1,2, \cdots, 8\}$. These $l_{i}$ are abtained in this manner

$$
\begin{aligned}
& l_{1}=\tilde{K}_{\alpha}^{L} \mathrm{~d} t+\tilde{H}_{\alpha}^{L} \mathrm{~d}\left(\tilde{B}_{t}\right)_{\alpha}^{L}, l_{2}=\tilde{K}_{\alpha}^{L} \mathrm{~d} t+\tilde{H}_{\alpha}^{L} \mathrm{~d}\left(\tilde{B}_{t}\right)_{\alpha}^{U}, \\
& l_{3}=\tilde{K}_{\alpha}^{L} \mathrm{~d} t+\tilde{H}_{\alpha}^{U} \mathrm{~d}\left(\tilde{B}_{t}\right)_{\alpha}^{L}, l_{4}=\tilde{K}_{\alpha}^{L} \mathrm{~d} t+\tilde{H}_{\alpha}^{U} \mathrm{~d}\left(\tilde{B}_{t}\right)_{\alpha}^{U},
\end{aligned}
$$




$$
\begin{aligned}
& l_{5}=\tilde{K}_{\alpha}^{U} \mathrm{~d} t+\tilde{H}_{\alpha}^{L} \mathrm{~d}\left(\tilde{B}_{t}\right)_{\alpha}^{L}, l_{6}=\tilde{K}_{\alpha}^{U} \mathrm{~d} t+\tilde{H}_{\alpha}^{L} \mathrm{~d}\left(\tilde{B}_{t}\right)_{\alpha}^{U}, \\
& l_{7}=\tilde{K}_{\alpha}^{2} \mathrm{~d} t+\tilde{H}_{\alpha}^{U} \mathrm{~d}\left(\tilde{B}_{t}\right)_{\alpha}^{L}, l_{8}=\tilde{K}_{\alpha}^{2} \mathrm{~d} t+\tilde{H}_{\alpha}^{U} \mathrm{~d}\left(\tilde{B}_{t}\right)_{\alpha}^{U} .
\end{aligned}
$$

Moreover,

$$
\left[\tilde{Y}_{t}\right]_{\alpha}=\left[\tilde{G}_{\alpha}^{L}\left(t,\left(\tilde{X}_{t}\right)_{\alpha}^{L},\left(\tilde{X}_{t}\right)_{\alpha}^{U}\right), \tilde{G}_{\alpha}^{U}\left(t,\left(\tilde{X}_{t}\right)_{\alpha}^{L},\left(\tilde{X}_{t}\right)_{\alpha}^{U}\right)\right],
$$

where

$$
\tilde{G}_{\alpha}^{L}\left(t,\left(\tilde{X}_{t}\right)_{\alpha}^{L},\left(\tilde{X}_{t}\right)_{\alpha}^{U}\right):=\min \left\{G(t, x): x \in\left[\left(\tilde{X}_{t}\right)_{\alpha}\right]\right\}
$$

and

$$
\tilde{G}_{\alpha}^{U}\left(t,\left(\tilde{X}_{t}\right)_{\alpha}^{L},\left(\tilde{X}_{t}\right)_{\alpha}^{U}\right):=\max \left\{G(t, x): x \in\left[\left(\tilde{X}_{t}\right)_{\alpha}\right]\right\}
$$

Without loss of generalities, we can deal with the case of the second order generalized Hukuhara differential of type (1) from the definition 2.4. We also consider that the domain $\mathbb{D}=\Omega \times[0, T]$ does not have a hinge point. Thus, we have the following expressions

$$
\begin{aligned}
& \partial_{t} \tilde{G}_{\alpha}^{L}\left(t,\left(\tilde{X}_{t}\right)_{\alpha}^{L},\left(\tilde{X}_{t}\right)_{\alpha}^{U}\right):=\min \left\{\partial_{t} G(t, x): x \in\left[\left(\tilde{X}_{t}\right)_{\alpha}\right]\right\}, \\
& \partial_{t} \tilde{G}_{\alpha}^{U}\left(t,\left(\tilde{X}_{t}\right)_{\alpha}^{L},\left(\tilde{X}_{t}\right)_{\alpha}^{U}\right):=\max \left\{\partial_{t} G(t, x): x \in\left[\left(\tilde{X}_{t}\right)_{\alpha}\right]\right\}, \\
& \partial_{x} \tilde{G}_{\alpha}^{U}\left(t,\left(\tilde{X}_{t}\right)_{\alpha}^{L},\left(\tilde{X}_{t}\right)_{\alpha}^{U}\right):=\min \left\{\partial_{x} G(t, x): x \in\left[\left(\tilde{X}_{t}\right)_{\alpha}\right]\right\}, \\
& \partial_{x} \tilde{G}_{\alpha}^{U}\left(t,\left(\tilde{X}_{t}\right)_{\alpha}^{L},\left(\tilde{X}_{t}\right)_{\alpha}^{U}\right):=\max \left\{\partial_{x} G(t, x): x \in\left[\left(\tilde{X}_{t}\right)_{\alpha}\right]\right\}, \\
& \partial_{x x} \tilde{G}_{\alpha}^{U}\left(t,\left(\tilde{X}_{t}\right)_{\alpha}^{L},\left(\tilde{X}_{t}\right)_{\alpha}^{U}\right):=\min \left\{\partial_{x x} G(t, x): x \in\left[\left(\tilde{X}_{t}\right)_{\alpha}\right]\right\}, \\
& \partial_{x x} \tilde{G}_{\alpha}^{U}\left(t,\left(\tilde{X}_{t}\right)_{\alpha}^{L},\left(\tilde{X}_{t}\right)_{\alpha}^{U}\right):=\max \left\{\partial_{x x} G\left(t, X_{t}\right): X_{t} \in\left[\left(\tilde{X}_{t}\right)_{\alpha}\right]\right\} .
\end{aligned}
$$

Hence, for all $X_{t} \in\left[\left(\tilde{X}_{t}\right)_{\alpha}\right]$ with $\alpha \in[0,1]$, from the classical It $\tilde{A}^{\prime}$ formula applied to the function $Y_{t}=G\left(t, X_{t}\right)$, we thus obtain

$$
\mathrm{d} Y_{t}=\frac{\partial G\left(t, X_{t}\right)}{\partial t} \mathrm{~d} t+\frac{\partial G\left(t, X_{t}\right)}{\partial x} \mathrm{~d} X_{t}+\frac{1}{2} \frac{\partial^{2} G\left(t, X_{t}\right)}{\partial x^{2}}\left(\mathrm{~d} X_{t}\right)^{2} .
$$

By taking the maximum and the minimum to every terms from the Equation (4.22) and from the Equations (4.16)-(4.21) we get

$$
\begin{aligned}
\mathrm{d}\left(\tilde{Y}_{t}\right)_{\alpha}^{L}= & \frac{\partial \tilde{G}_{\alpha}^{L}\left(t,\left(\tilde{X}_{t}\right)_{\alpha}^{L},\left(\tilde{X}_{t}\right)_{\alpha}^{U}\right)}{\partial t} \mathrm{~d} t+\frac{\partial \tilde{G}_{\alpha}^{L}\left(t,\left(\tilde{X}_{t}\right)_{\alpha}^{L},\left(\tilde{X}_{t}\right)_{\alpha}^{U}\right)}{\partial x} \mathrm{~d}\left(\tilde{X}_{t}\right)_{\alpha}^{L} \\
& +\frac{1}{2} \frac{\partial^{2} \tilde{G}_{\alpha}^{L}\left(t,\left(\tilde{X}_{t}\right)_{\alpha}^{L},\left(\tilde{X}_{t}\right)_{\alpha}^{U}\right)}{\partial x^{2}}\left(\mathrm{~d}\left(\tilde{X}_{t}\right)_{\alpha}^{L}\right)^{2}
\end{aligned}
$$

and

$$
\begin{aligned}
\mathrm{d}\left(\tilde{Y}_{t}\right)_{\alpha}^{U}= & \frac{\partial \tilde{G}_{\alpha}^{U}\left(t,\left(\tilde{X}_{t}\right)_{\alpha}^{L},\left(\tilde{X}_{t}\right)_{\alpha}^{U}\right)}{\partial t} \mathrm{~d} t+\frac{\partial \tilde{G}_{\alpha}^{U}\left(t,\left(\tilde{X}_{t}\right)_{\alpha}^{L},\left(\tilde{X}_{t}\right)_{\alpha}^{U}\right)}{\partial x} \mathrm{~d}\left(\tilde{X}_{t}\right)_{\alpha}^{U} \\
& +\frac{1}{2} \frac{\partial^{2} \tilde{G}_{\alpha}^{U}\left(t,\left(\tilde{X}_{t}\right)_{\alpha}^{L},\left(\tilde{X}_{t}\right)_{\alpha}^{U}\right)}{\partial x^{2}}\left(\mathrm{~d}\left(\tilde{X}_{t}\right)_{\alpha}^{U}\right)^{2} .
\end{aligned}
$$


Then, we have

$$
\begin{aligned}
& {\left[\mathrm{d}\left(\tilde{Y}_{t}\right)_{\alpha}^{L}, \mathrm{~d}\left(\tilde{Y}_{t}\right)_{\alpha}^{U}\right]=\left[\frac{\partial \tilde{G}_{\alpha}^{L}\left(t,\left(\tilde{X}_{t}\right)_{\alpha}^{L},\left(\tilde{X}_{t}\right)_{\alpha}^{U}\right)}{\partial t} \mathrm{~d} t, \frac{\partial \tilde{G}_{\alpha}^{U}\left(t,\left(\tilde{X}_{t}\right)_{\alpha}^{L},\left(\tilde{X}_{t}\right)_{\alpha}^{U}\right)}{\partial t} \mathrm{~d} t\right]} \\
& \oplus_{\text {int }}\left[\frac{\partial \tilde{G}_{\alpha}^{L}\left(t,\left(\tilde{X}_{t}\right)_{\alpha}^{L},\left(\tilde{X}_{t}\right)_{\alpha}^{U}\right)}{\partial x} \mathrm{~d}\left(\tilde{X}_{t}\right)_{\alpha}^{L}, \frac{\partial \tilde{G}_{\alpha}^{U}\left(t,\left(\tilde{X}_{t}\right)_{\alpha}^{L},\left(\tilde{X}_{t}\right)_{\alpha}^{U}\right)}{\partial x} \mathrm{~d}\left(\tilde{X}_{t}\right)_{\alpha}^{U}\right] \\
& \oplus_{\text {int }}\left[\frac{1}{2} \frac{\partial^{2} \tilde{G}_{\alpha}^{L}\left(t,\left(\tilde{X}_{t}\right)_{\alpha}^{L},\left(\tilde{X}_{t}\right)_{\alpha}^{U}\right)}{\partial x^{2}}\left(\mathrm{~d}\left(\tilde{X}_{t}\right)_{\alpha}^{L}\right)^{2}, \frac{1}{2} \frac{\partial^{2} \tilde{G}_{\alpha}^{U}\left(t,\left(\tilde{X}_{t}\right)_{\alpha}^{L},\left(\tilde{X}_{t}\right)_{\alpha}^{U}\right)}{\partial x^{2}}\left(\mathrm{~d}\left(\tilde{X}_{t}\right)_{\alpha}^{U}\right)^{2}\right]
\end{aligned}
$$

Because all the derivatives are taken in the sense of generalized Hukuara and due to the definition 2.4, we have for every $\alpha \in[0,1]$

$$
\begin{aligned}
{\left[\mathrm{d} \tilde{Y}_{t}\right]_{\alpha}=} & {\left[\partial_{t_{g H}} \tilde{G}\left(t, \tilde{X}_{t}\right)\right]_{\alpha} \mathrm{d} t \oplus_{i n t}\left[\partial_{x_{g H}} \tilde{G}\left(t, \tilde{X}_{t}\right) \mathrm{d} \tilde{X}_{t}\right]_{\alpha} } \\
& \oplus_{i n t} \frac{1}{2} \odot_{i n t}\left[\partial_{x x_{g H}} \tilde{G}\left(t, \tilde{X}_{t}\right) \otimes\left(\mathrm{d} \tilde{X}_{t}\right)^{2}\right]_{\alpha} .
\end{aligned}
$$

Thus we obtain for every $\alpha \in[0,1]$

$$
\left[\mathrm{d} \tilde{Y}_{t}\right]_{\alpha}=\left[\partial_{t_{g H}} \tilde{G}\left(t, \tilde{X}_{t}\right) \mathrm{d} t \oplus \partial_{x_{g H}} \tilde{G}\left(t, \tilde{X}_{t}\right) \otimes \mathrm{d} \tilde{X}_{t} \oplus \frac{1}{2} \odot \partial_{x x_{g H}} \tilde{G}\left(t, \tilde{X}_{t}\right) \otimes\left(\mathrm{d} \tilde{X}_{t}\right)^{2}\right]_{\alpha} .
$$

Remark 4.3. The general case should take into account that the domain would just contain some hinge points. This case could be reduced to the treated case by subdividing the domain in several areas in which we do not have the hinge point inside but only at the edges of these subdomains. Some properties are useful in the following.

Theorem 4.4. Let $\{\tilde{X}(t)\}_{t \geq 0},\{\tilde{Y}(t)\}_{t \geq 0} \in \mathcal{L}^{2}\left(\mathbb{F}_{c}(\mathbb{R})\right)$. Then, we have

$$
E\left[D^{2}\left(\int_{0}^{t} \tilde{X}(s) \mathrm{d} \tilde{B}_{s}, \int_{0}^{t} \tilde{Y}(s) \mathrm{d} \tilde{B}_{s}\right)\right] \leq E\left[\int_{0}^{t} D^{2}(\tilde{X}(s), \tilde{Y}(s)) \mathrm{d} s\right] .
$$

Proof. From the definition of $D$, we have for all $t \geq 0$

$$
\begin{aligned}
& E\left[D^{2}\left(\int_{0}^{t} \tilde{X}(s) \mathrm{d} \tilde{B}_{s}, \int_{0}^{t} \tilde{Y}(s) \mathrm{d} \tilde{B}_{s}\right)\right] \\
& =E\left[\sup _{\alpha \in[0,1]} d_{H}^{2}\left(\left[\int_{0}^{t} \tilde{X}(s) \mathrm{d} \tilde{B}_{s}\right]_{\alpha},\left[\int_{0}^{t} \tilde{Y}(s) \mathrm{d} \tilde{B}_{s}\right]_{\alpha}\right)\right] \\
& \leq E\left[\sup _{\alpha \in[0,1]} d_{H}^{2}\left(\int_{0}^{t} \tilde{X}_{\alpha}(s) \mathrm{d} \tilde{B}_{\alpha}^{U}(s), \int_{0}^{t} \tilde{Y}_{\alpha}(s) \mathrm{d} \tilde{B}_{\alpha}^{U}(s)\right)\right] \\
& \leq E\left[\sup _{\alpha \in[0,1]} \int_{0}^{t} d_{H}^{2}\left(\tilde{X}_{\alpha}(s), \tilde{Y}_{\alpha}(s)\right) \mathrm{d} s\right] \\
& \leq E\left[\int_{0}^{t} \sup _{\alpha \in[0,1]} d_{H}^{2}\left(\tilde{X}_{\alpha}(s), \tilde{Y}_{\alpha}(s)\right) \mathrm{d} s\right] \\
& =E\left[\int_{0}^{t} D^{2}(\tilde{X}(s), \tilde{Y}(s)) \mathrm{d} s\right] .
\end{aligned}
$$

We have expanded out and have assumed that $\mathrm{d} \tilde{B}_{\alpha}(s)=\left[\mathrm{d} \tilde{B}_{\alpha}^{L}(s), \mathrm{d} \tilde{B}_{\alpha}^{U}(s)\right]$. 


\section{Fuzzy Stochastic Differential Equations}

Notice that the notion of fuzzy differential equations defined in an uncertain framework has already been the subject of several investigations (see Buckley and Feuring [17] and Puri and Ralescu [2]). In the same way, we will define in this section, the notion of fuzzy stochastic differential equations driven by a fuzzy Brownian motion. Using the Hukuhara differential, we have the following definition.

Definition 5.1. A fuzzy stochastic differential Equation (FSDE), on a filtered probability space $\left(\Omega, \mathcal{F},\{\mathcal{F}\}_{t \geq 0}, P\right)$ and driven by a fuzzy Brownian motion is given by

$$
\tilde{X}_{0}=\tilde{x}, \quad \mathrm{~d} \tilde{X}_{t}=\tilde{F}\left(t, \tilde{X}_{t}\right) \mathrm{d} t \oplus \tilde{G}\left(t, \tilde{X}_{t}\right) \mathrm{d} \tilde{B}_{t},
$$

where $\tilde{F}$ and $\tilde{G}$ are borel-measurable fuzzy stochastic functions defined from $[0, \infty) \times \mathbb{F}_{c}(\mathbb{R})$ with values in $\mathbb{F}_{c}(\mathbb{R})$.

In the integral form, the fuzzy stochastic differential Equation (FSDE) driven by a fuzzy Brownian motion (5.1) is denoted

$$
\tilde{X}_{t}=\tilde{X}_{0} \oplus \int_{0}^{t} \tilde{F}\left(s, \tilde{X}_{s}\right) \mathrm{d} s \oplus \int_{0}^{t} \tilde{G}\left(s, \tilde{X}_{s}\right) \mathrm{d} \tilde{B}_{s} .
$$

The solution of (5.1) is defined like that:

Definition 5.2. A solution of a fuzzy stochastic differential Equation (FSDE) driven by a fuzzy Brownian motion (5.1) is a fuzzy stochastic process $\left\{\tilde{X}_{t}\right\}_{t \geq 0}$ defined on $\left(\Omega, \mathcal{F},\{\mathcal{F}\}_{t \geq 0}, P\right)$ such that:

1) There exists a one-dimensional fuzzy Brownian motion, $\left\{\tilde{B}_{t}\right\}_{t \geq 0}$ with $\tilde{B}_{0}=1_{\{0\}} \quad P$-a.e;

2) $\left\{\tilde{X}_{t}\right\}_{t \geq 0}$ is $\mathcal{F}_{t}$-adapted and continuous in $t P$-a.e, that is $D(\tilde{X}(t+h), \tilde{X}(t)) \rightarrow 0$ for $h \rightarrow 0$ with probability 1 ;

3) $\left\{\tilde{X}_{t}\right\}_{t \geq 0}$ et $\left\{\tilde{B}_{t}\right\}_{t \geq 0}$ satisfy (5.2).

Definition 5.3. There is uniqueness of a trajectory if for any probability space $(\Omega, \mathcal{F}, P)$, every filtration $\{\mathcal{F}\}_{t \geq 0}$ and every fuzzy Brownian motion $\left\{\tilde{B}_{t}\right\}_{t \geq 0}$, two fuzzy solutions $\tilde{X}_{t}$ and $\tilde{Y}_{t}$ such that $\tilde{X}_{0}=\tilde{Y}_{0}$ almost everywhere, verifies almost everywhere

$$
\tilde{X}_{t}=\tilde{Y}_{t}, \quad \forall t \geq 0 .
$$

We say that $\tilde{X}_{t}$ is a strong fuzzy solution wether $\tilde{X}_{t}$ is adapted to the canonical fuzzy filtration $\left(\sigma\left(\tilde{B}_{t}: s \leq t\right)\right)_{t \geq 0}$.

There is a weak unicity since two solutions of the fuzzy stochastic differential equation have necessarily the same law.

Theorem 5.4. Let $\left\{\tilde{X}_{t}\right\}_{t>0}$ and $\left\{\tilde{Y}_{t}\right\}_{t>0}$ in $\mathcal{L}_{2}\left(\mathbb{F}_{c}(\mathbb{R})\right)$ such that

$$
\left(\int_{0}^{t} \tilde{X}_{\alpha}^{U}(s) \mathrm{d} \tilde{B}_{\alpha}^{U}(s)-\int_{0}^{t} \tilde{X}_{\alpha}^{L}(s) \mathrm{d} \tilde{B}_{\alpha}^{L}(s)\right)\left(\int_{0}^{t} \tilde{Y}_{\alpha}^{U}(s) \mathrm{d} \tilde{B}_{\alpha}^{U}(s)-\int_{0}^{t} \tilde{Y}_{\alpha}^{L}(s) \mathrm{d} \tilde{B}_{\alpha}^{L}(s)\right) \geq 0
$$

or 


$$
\left(\int_{0}^{t} \tilde{X}_{\alpha}^{U}(s) \mathrm{d} \tilde{B}_{\alpha}^{L}(s)-\int_{0}^{t} \tilde{X}_{\alpha}^{L}(s) \mathrm{d} \tilde{B}_{\alpha}^{U}(s)\right)\left(\int_{0}^{t} \tilde{Y}_{\alpha}^{U}(s) \mathrm{d} \tilde{B}_{\alpha}^{L}(s)-\int_{0}^{t} \tilde{Y}_{\alpha}^{L}(s) \mathrm{d} \tilde{B}_{\alpha}^{U}(s)\right) \geq 0
$$

for all $\alpha \in[0,1]$ and $t \in[0, T]$ a.s.. Then for all $t \in[0, T]$,

$$
E\left[\sup _{0 \leq u \leq t} D^{2}\left(\int_{0}^{u} \tilde{X}(s) \mathrm{d} \tilde{B}_{s}, \int_{0}^{u} \tilde{Y}(s) \mathrm{d} \tilde{B}_{s}\right)\right] \leq 4 E\left[\int_{0}^{t} D^{2}(\tilde{X}(s), \tilde{Y}(s)) \mathrm{d} s\right] .
$$

Proof. Due to the Doob inequality and the classical Itô integral isometry we obtain from the hypothesis (5.3)

$$
\begin{aligned}
& E\left[\sup _{0 \leq u \leq t} D^{2}\left(\int_{0}^{u} \tilde{X}(s) \mathrm{d} \tilde{B}_{s}, \int_{0}^{u} \tilde{Y}(s) \mathrm{d} \tilde{B}_{s}\right)\right] \\
& =E\left[\sup _{0 \leq u \leq t} \sup _{0 \leq \alpha \leq 1} d_{H}^{2}\left(\left[\int_{0}^{u} \tilde{X}(s) \mathrm{d} \tilde{B}_{s}\right]_{\alpha},\left[\int_{0}^{u} \tilde{Y}(s) \mathrm{d} \tilde{B}_{s}\right]_{\alpha}\right)\right] \\
& =E\left[\operatorname { s u p } _ { 0 \leq u \leq t } \operatorname { s u p } _ { 0 \leq \alpha \leq 1 } \operatorname { m a x } \left\{\left|\int_{0}^{u} \tilde{X}_{\alpha}^{L}(s) \mathrm{d} \tilde{B}_{\alpha}^{L}(s)-\int_{0}^{u} \tilde{Y}_{\alpha}^{L}(s) \mathrm{d} \tilde{B}_{\alpha}^{L}(s)\right|^{2},\right.\right. \\
& \left.\left.\left|\int_{0}^{u} \tilde{X}_{\alpha}^{U}(s) \mathrm{d} \tilde{B}_{\alpha}^{U}(s)-\int_{0}^{u} \tilde{Y}_{\alpha}^{U}(s) \mathrm{d} \tilde{B}_{\alpha}^{U}(s)\right|^{2}\right\}\right] \\
& =E\left[\sup _{0 \leq u \leq t} \sup _{0 \leq \alpha \leq 1} \max \left\{\left|\int_{0}^{u} \tilde{X}_{\alpha}^{L}(s)-\tilde{Y}_{\alpha}^{L}(s) \mathrm{d} \tilde{B}_{\alpha}^{L}(s)\right|^{2},\left|\int_{0}^{u} \tilde{X}_{\alpha}^{U}(s)-\tilde{Y}_{\alpha}^{U}(s) \mathrm{d} \tilde{B}_{\alpha}^{U}(s)\right|^{2}\right\}\right] \\
& \leq 4 E\left[\sup _{0 \leq \alpha \leq 1} \max \left\{\left|\int_{0}^{t} \tilde{X}_{\alpha}^{L}(s)-\tilde{Y}_{\alpha}^{L}(s) \mathrm{d} \tilde{B}_{\alpha}^{L}(s)\right|^{2},\left|\int_{0}^{t} \tilde{X}_{\alpha}^{U}(s)-\tilde{Y}_{\alpha}^{U}(s) \mathrm{d} \tilde{B}_{\alpha}^{U}(s)\right|^{2}\right\}\right] \\
& \leq 4 E\left[\int_{0}^{t} \sup _{0 \leq \alpha \leq 1} \max \left\{\left|\tilde{X}_{\alpha}^{L}(s)-\tilde{Y}_{\alpha}^{L}(s)\right|^{2},\left|\tilde{X}_{\alpha}^{U}(s)-\tilde{Y}_{\alpha}^{U}(s)\right|^{2}\right\} \mathrm{d} s\right] \\
& =4 E\left[\int_{0}^{t} D^{2}(\tilde{X}(s), \tilde{Y}(s)) \mathrm{d} s\right] \text {. } \\
& \text { And from the hypothesis (5.4), we get } \\
& E\left[\sup _{0 \leq u \leq t} D^{2}\left(\int_{0}^{u} \tilde{X}(s) \mathrm{d} \tilde{B}_{s}, \int_{0}^{u} \tilde{Y}(s) \mathrm{d} \tilde{B}_{s}\right)\right] \\
& =E\left[\sup _{0 \leq u \leq t} \sup _{0 \leq \alpha \leq 1} d_{H}^{2}\left(\left[\int_{0}^{u} \tilde{X}(s) \mathrm{d} \tilde{B}_{s}\right]_{\alpha},\left[\int_{0}^{u} \tilde{Y}(s) \mathrm{d} \tilde{B}_{s}\right]_{\alpha}\right)\right] \\
& =E\left[\operatorname { s u p } _ { 0 \leq u \leq t } \operatorname { s u p } _ { 0 \leq \alpha \leq 1 } \operatorname { m a x } \left\{\left|\int_{0}^{u} \tilde{X}_{\alpha}^{L}(s) \mathrm{d} \tilde{B}_{\alpha}^{U}(s)-\int_{0}^{u} \tilde{Y}_{\alpha}^{L}(s) \mathrm{d} \tilde{B}_{\alpha}^{U}(s)\right|^{2}\right.\right. \text {, } \\
& \left.\left.\left|\int_{0}^{u} \tilde{X}_{\alpha}^{U}(s) \mathrm{d} \tilde{B}_{\alpha}^{L}(s)-\int_{0}^{u} \tilde{Y}_{\alpha}^{U}(s) \mathrm{d} \tilde{B}_{\alpha}^{L}(s)\right|^{2}\right\}\right] \\
& =E\left[\sup _{0 \leq u \leq t} \sup _{0 \leq \alpha \leq 1} \max \left\{\left|\int_{0}^{u} \tilde{X}_{\alpha}^{L}(s)-\tilde{Y}_{\alpha}^{L}(s) \mathrm{d} \tilde{B}_{\alpha}^{U}(s)\right|^{2},\left|\int_{0}^{u} \tilde{X}_{\alpha}^{U}(s)-\tilde{Y}_{\alpha}^{U}(s) \mathrm{d} \tilde{B}_{\alpha}^{L}(s)\right|^{2}\right\}\right] \\
& \leq 4 E\left[\sup _{0 \leq \alpha \leq 1} \max \left\{\left|\int_{0}^{t} \tilde{X}_{\alpha}^{L}(s)-\tilde{Y}_{\alpha}^{L}(s) \mathrm{d} \tilde{B}_{\alpha}^{U}(s)\right|^{2},\left|\int_{0}^{t} \tilde{X}_{\alpha}^{U}(s)-\tilde{Y}_{\alpha}^{U}(s) \mathrm{d} \tilde{B}_{\alpha}^{L}(s)\right|^{2}\right\}\right] \\
& \leq 4 E\left[\int_{0}^{t} \sup _{0 \leq \alpha \leq 1} \max \left\{\left|\tilde{X}_{\alpha}^{L}(s)-\tilde{Y}_{\alpha}^{L}(s)\right|^{2},\left|\tilde{X}_{\alpha}^{U}(s)-\tilde{Y}_{\alpha}^{U}(s)\right|^{2}\right\} \mathrm{d} s\right] \\
& =4 E\left[\int_{0}^{t} D^{2}(\tilde{X}(s), \tilde{Y}(s)) \mathrm{d} s\right] \text {. }
\end{aligned}
$$

Before stating and proving the existence and the uniqueness theorem of solutions of a FSDE, let us present a version of the Gronwall Lemma that will help us 
to prove this theorem.

Lemma 5.5. (Gronwall Lemma, Bouleau [18], Lamberton and Lapeyre [19]) if $f$ is a continuous function, such that for all $0 \leq t \leq T$, we have $f(t) \leq a+b \int_{0}^{t} f(s) \mathrm{d} s$, then $f(T) \leq a\left(1+\mathrm{e}^{b T}\right)$.

We can now state and prove the following important theorem:

Theorem 5.6. Assume that the fuzzy functions $\tilde{F}:[0, \infty) \times \mathbb{F}_{c}(\mathbb{R}) \mapsto \mathbb{F}_{c}(\mathbb{R})$ and $\tilde{G}:[0, \infty) \times \mathbb{F}_{c}(\mathbb{R}) \mapsto \mathbb{F}_{c}(\mathbb{R})$ satisfy the following conditions:

1) There exists a constant $K>0$ such that

$$
\begin{gathered}
D^{2}(\tilde{F}(t, \tilde{x}), \tilde{F}(t, \tilde{y}))+D^{2}(\tilde{G}(t, \tilde{x}), \tilde{G}(t, \tilde{y})) \leq K D^{2}(\tilde{x}, \tilde{y}), \\
\|\|[\tilde{F}(t, x)]^{0}\|\mid\|\left\|[\tilde{G}(t, x)]^{0}\right\| \leq K\left(1+\left\|[\tilde{x}]^{0}\right\| \|\right)
\end{gathered}
$$

for every $\tilde{x} \in \mathbb{F}_{c}(\mathbb{R})$ and $t \in[0, \infty)$;

2) For all fuzzy stochastic processes $(\tilde{Y}(t))_{t \geq 0} \in \mathcal{L}^{2}\left(\mathbb{F}_{c}(\mathbb{R})\right)$, the fuzzy stochastic process $(\tilde{G}(t, \tilde{Y}(t)))_{t \geq 0}$ has the parametric representation $\left([\tilde{G}(\cdot, \tilde{Y}(\cdot))]_{\alpha}(t)\right)_{t \geq 0}$ defined by

$$
[\tilde{G}(\cdot, \tilde{Y}(\cdot))]_{\alpha}(t)=\left[\tilde{G}_{\alpha}^{L}\left(t, \tilde{Y}_{\alpha}^{L}(t), \tilde{Y}_{\alpha}^{U}(t)\right), \tilde{G}_{\alpha}^{L}\left(t, \tilde{Y}_{\alpha}^{L}(t), \tilde{Y}_{\alpha}^{U}(t)\right)\right] ;
$$

3) For all $\left\{\tilde{Y}_{1}(t)\right\}_{t \geq 0}$ et $\left\{\tilde{Y}_{2}(t)\right\}_{t \geq 0}$ in $\mathcal{L}_{2}\left(\mathbb{F}_{c}(\mathbb{R})\right)$, we assume that

$$
\begin{aligned}
& \left(\int_{0}^{t}\left(\tilde{Y}_{1}\right)_{\alpha}^{U}(s) \mathrm{d} \tilde{B}_{\alpha}^{U}(s)-\int_{0}^{t}\left(\tilde{Y}_{1}\right)_{\alpha}^{L}(s) \mathrm{d} \tilde{B}_{\alpha}^{L}(s)\right) \\
& \times\left(\int_{0}^{t}\left(\tilde{Y}_{2}\right)_{\alpha}^{U}(s) \mathrm{d} \tilde{B}_{\alpha}^{U}(s)-\int_{0}^{t}\left(\tilde{Y}_{2}\right)_{\alpha}^{L}(s) \mathrm{d} \tilde{B}_{\alpha}^{L}(s)\right) \geq 0
\end{aligned}
$$

or

$$
\begin{aligned}
& \left(\int_{0}^{t}\left(\tilde{Y}_{1}\right)_{\alpha}^{U}(s) \mathrm{d} \tilde{B}_{\alpha}^{L}(s)-\int_{0}^{t}\left(\tilde{Y}_{1}\right)_{\alpha}^{L}(s) \mathrm{d} \tilde{B}_{\alpha}^{U}(s)\right) \\
& \times\left(\int_{0}^{t}\left(\tilde{Y}_{2}\right)_{\alpha}^{U}(s) \mathrm{d} \tilde{B}_{\alpha}^{L}(s)-\int_{0}^{t}\left(\tilde{Y}_{2}\right)_{\alpha}^{L}(s) \mathrm{d} \tilde{B}_{\alpha}^{U}(s)\right) \geq 0
\end{aligned}
$$

for all $t \in[0, T]$ a.e.

Then the fuzzy stochastic differential equation driven by a fuzzy Brownian motion (5.1) admits a unique solution.

Proof. Let $T>0$, for all $t \in[0, T]$, we define $\tilde{X}_{0}(t)=\tilde{x}_{0}$. Then from the condition (1) of the theorem, we get

$$
E\left[\int_{0}^{T}\|\|\left[\tilde{G}\left(t, \tilde{x}_{0}\right)\right]^{0}\|\|^{2} \mathrm{~d} t\right] \leq T K\left(1+E\left[\|\|\left[\tilde{x}_{0}\right]^{0} \|\left.^{2}\right|^{2}\right]\right)<\infty
$$

and thus, $\left\{\tilde{G}\left(t, \tilde{x}_{0}\right)\right\}_{t \geq 0} \in \mathcal{L}^{2}\left(\mathbb{F}_{c}(\mathbb{R})\right)$. From the definition of the fuzzy stochastic integral with respect to a fuzzy brownian motion, $\int_{0}^{t} \tilde{G}\left(s, \tilde{x}_{0}\right) \mathrm{d} \tilde{B}_{s}$ is well-defined. So we can define a continuous fuzzy stochastic process

$$
\tilde{X}_{1}(t)=\tilde{x}_{0} \oplus \int_{0}^{t} \tilde{F}\left(s, \tilde{x}_{0}\right) \mathrm{d} s \oplus \int_{0}^{t} \tilde{G}\left(s, \tilde{x}_{0}\right) \mathrm{d} \tilde{B}_{s}
$$

with $E\left[\|\|\left[\tilde{X}_{1}(t)\right]^{0} \|\left.^{2}\right|^{2}\right]<\infty$, and that for all $t \in[0, T]$. Now suppose that fuzzy 
continuous processes

$$
\tilde{X}_{i}(t)=\tilde{x}_{0} \oplus \int_{0}^{t} \tilde{F}\left(s, \tilde{X}_{i-1}(s)\right) \mathrm{d} s \oplus \int_{0}^{t} \tilde{G}\left(s, \tilde{X}_{i-1}(s)\right) \mathrm{d} \tilde{B}_{s}, \quad i=2,3, \cdots, n,
$$

are defined and verify $\sup _{0 \leq t \leq T} E\left[\|\|\left[\tilde{X}_{i}(t)\right]^{0} \|\left.^{2}\right|^{2}\right]<\infty$. So by the condition (1) of the theorem, we get

$$
E\left[\int_{0}^{t}\left\|\left[\tilde{G}\left(s, \tilde{X}_{n}(s)\right)\right]^{0}\right\|^{2} \|^{\mathrm{d} s}\right] \leq T K\left(1+\sup _{0 \leq t \leq T} E\left[\left\|\left[\tilde{X}_{i}(t)\right]^{0}\right\| \|^{2}\right]\right)<\infty
$$

and then, $\left\{\tilde{G}\left(t, \tilde{X}_{n}(t)\right)\right\}_{t>0} \in \mathcal{L}^{2}\left(\mathbb{F}_{c}(\mathbb{R})\right)$.

Which shows the fuzzy Itô integral $\int_{0}^{t} \tilde{G}\left(s, \tilde{X}_{n}(s)\right) \mathrm{d} \tilde{B}_{s}$ is well-defined. Hence we can define a continuous fuzzy stochastic process

$$
\tilde{X}_{n+1}(t)=\tilde{x}_{0} \oplus \int_{0}^{t} \tilde{F}\left(s, \tilde{X}_{n}(s)\right) \mathrm{d} s \oplus \int_{0}^{t} \tilde{G}\left(s, \tilde{X}_{n}(s)\right) \mathrm{d} \tilde{B}_{s} .
$$

From mathematical induction, we obtain a sequence of fuzzy stochastic processes $\left\{\left\{\tilde{X}_{n}(t)\right\}_{t \geq 0}\right\}, n=1,2, \cdots$ in $\mathcal{L}^{2}\left(\mathbb{F}_{c}(\mathbb{R})\right)$. Due to the Theorems 4.4 and 5.4 , and from the equalities (2) and (4) of the Theorem and under our hypothesis, we get

$$
\begin{aligned}
& E\left[\sup _{0 \leq t \leq T} D^{2}\left(\tilde{X}_{n}(t), \tilde{X}_{n+1}(t)\right)\right] \\
& \leq 2 E\left[\sup _{0 \leq t \leq T} D^{2}\left(\int_{0}^{t} \tilde{F}\left(s, \tilde{X}_{n-1}(s)\right) \mathrm{d} s, \int_{0}^{t} \tilde{F}\left(s, \tilde{X}_{n}(s)\right) \mathrm{d} s\right)\right] \\
& +2 E\left[\sup _{0 \leq t \leq T} D^{2}\left(\int_{0}^{t} \tilde{G}\left(s, \tilde{X}_{n-1}(s)\right) \mathrm{d} \tilde{B}_{s}, \int_{0}^{t} \tilde{G}\left(s, \tilde{X}_{n}(s)\right) \mathrm{d} \tilde{B}_{s}\right)\right] \\
& \leq 2 T E\left[\int_{0}^{T} D^{2}\left(\tilde{F}\left(s, \tilde{X}_{n-1}(s)\right), \tilde{F}\left(s, \tilde{X}_{n}(s)\right)\right) \mathrm{d} s\right] \\
& +8 E\left[\int_{0}^{T} D^{2}\left(\tilde{G}\left(s, \tilde{X}_{n-1}(s)\right), \tilde{G}\left(s, \tilde{X}_{n}(s)\right)\right) \mathrm{d} s\right] \\
& \leq(2 T+8) K \int_{0}^{T} E\left[\sup _{0 \leq s \leq t} D^{2}\left(\tilde{X}_{n-1}(s), \tilde{X}_{n}(s)\right)\right] \mathrm{d} t \\
& \leq K_{T}^{n} \int_{0}^{T} \int_{0}^{t_{1}} \cdots \int_{0}^{t_{n-1}} E\left[\sup _{0 \leq s \leq t_{n}} D^{2}\left(\tilde{X}_{0}, \tilde{X}_{1}(s)\right)\right] \mathrm{d} t_{n} \cdots \mathrm{d} t_{2} \mathrm{~d} t_{1},
\end{aligned}
$$

where $K_{T}=(2 T+8) K$. From the same above reason, we get

$$
E\left[\sup _{0 \leq s \leq t_{n}} D^{2}\left(\tilde{x}_{0}, \tilde{X}_{1}(t)\right)\right] \leq K_{T}\left(1+\left\|\left[\tilde{x}_{0}\right]_{0}\right\| \|^{2}\right) .
$$

So we have

$$
E\left[\sup _{0 \leq t \leq T} D^{2}\left(\tilde{X}_{n}(t), \tilde{X}_{n+1}(t)\right)\right] \leq \frac{K_{T}^{n+1}}{(n+1) !}\left(1+\left\|[\tilde{x}]^{0}\right\|^{2}\right) .
$$

We get from the Chebyshev inequality

$$
\begin{aligned}
& P\left(\sup _{0 \leq t \leq T} D\left(\tilde{X}_{n}(t), \tilde{X}_{n+1}(t)\right) \geq \frac{1}{2^{n+1}}\right) \\
& \leq 4^{n+1} E\left[\sup _{0 \leq t \leq T} D^{2}\left(\tilde{X}_{n}(t), \tilde{X}_{n+1}(t)\right)\right] \leq C(T) \frac{K_{T}^{n+1}}{(n+1) !},
\end{aligned}
$$


where $C(T)>0$ is appropriately a constant that only depend on $\left\|\left[\tilde{x}_{0}\right]_{0}\right\|$ and $T$. From the fact that $\mathbb{F}_{c}(\mathbb{R})$ is a closed subspace of the metric space $(\mathbb{F}(\mathbb{R}), D)$ and from the Borel-Cantelli Lemma, we see that $\tilde{X}_{n}(t)$ uniformly converges with probability one on $[0, T] . T$ is arbitrary, $\tilde{X}(t)=\lim _{n \rightarrow \infty} \tilde{X}_{n}(t)$ determine a continuous fuzzy process that is clearly a solution of (5.1).

To show the unicity of the solution, let $\{\tilde{X}(t)\}_{t \geq 0}$ and $\left\{\tilde{X}^{\prime}(t)\right\}_{t \geq 0}$ two solutions of (5.1). So by above similar computations, we have

$$
E\left[D^{2}\left(\tilde{X}(t), \tilde{X}^{\prime}(t)\right)\right] \leq 2 K(1+T) \int_{0}^{t} E\left[D^{2}\left(\tilde{X}(s), \tilde{X}^{\prime}(s)\right)\right] \mathrm{d} s
$$

for all $t \in[0, T]$. We get from the Lemma 5.5 (Gronwall Lemma) that

$$
E\left[D^{2}\left(\tilde{X}(t), \tilde{X}^{\prime}(t)\right)\right]=0
$$

for all $t \in[0, T]$. Hence, for $T \rightarrow \infty$, we have $\tilde{X}(t)=\tilde{X}^{\prime}(t)$ almost everywhere for all $t \geq 0$. As $\{\tilde{X}(t)\}_{t \geq 0}$ and $\left\{\tilde{X}^{\prime}(t)\right\}_{t \geq 0}$ are D-continuous in $t$ almost everywhere, we can conclude that $\tilde{X}(t)=\tilde{X}^{\prime}(t)$ for all $t \geq 0$ almost everywhere.

\section{Conclusion}

The fuzzy stochastic differential equations treated in this paper constitute our great contribution in this field. We essentially based ourselves on the use of the Hukuhara differential by treating for certain cases of a single type of parametric representation between a fuzzy function and its different fuzzy Hukuhara derivatives. As an application of our results, we will soon treat the fuzzy OrnsteinUhlenbeck process and the fuzzy Geometric Brownian Motion. By applying the methods presented in this article, it would be possible to deal with the fuzzy version of Partial Derivative Equations of Posson and Klein-Gordon with Neumann conditions as a generalized Problem of Two-Dimensional.

\section{Conflicts of Interest}

The authors declare no conflicts of interest regarding the publication of this paper.

\section{References}

[1] Kumwimba, D., Mabela R., Rémon, M. and Walo, R. (2015) Fuzzy Itô Integral Driven by a Fuzzy Brownian Motion. Journal of Fuzzy Set Valued Analysis, 2015, 232-244.

[2] Puri, M.L. and Ralescu, D.A. (1983) Differentials of Fuzzy Functions. Journal of Mathematical Analysis and Applications, 91, 552-558. https://doi.org/10.1016/0022-247X(83)90169-5

[3] Kaleva, O. (1987) Fuzzy Differential Equations. Fuzzy Sets and Systems, 24, 301-317. https://doi.org/10.1016/0165-0114(87)90029-7

[4] Kaleva, O. (2006) A Note on Fuzzy Differential Equations. Nonlinear Analysis. Theory, Methods \& Applications, 64, 895-900. https://doi.org/10.1016/j.na.2005.01.003

[5] Malinowski, M.T. (2012) Strong Solutions to Stochastic Fuzzy Differential Equations of Itô Type. Mathematical and Computer Modelling, 55, 918-928. https://doi.org/10.1016/j.mcm.2011.09.018 
[6] Malinowski, M.T. (2012) Itô Type Stochastic Fuzzy Differential Equations with Delay. Systems \& Control Letters, 61, 692-701. https://doi.org/10.1016/j.sysconle.2012.02.012

[7] Malinowski, M.T. (2013) Some Properties of Strong Solutions to Stochastic Fuzzy Differential Equations. Information Sciences, 252, 62-80. https://doi.org/10.1016/j.ins.2013.02.053

[8] AbuAarqob, O.A., Shawagfeh, N.T. and AbuGhneim, O.A. (2008) Functions Defined on Fuzzy Real Numbers According to Zadeh's Extension. International Mathematical Forum, 3, 763-776.

[9] Allahviranloo, T., Gouyandeh, Z., Armand, A. and Hasanoglu, A. (2015) On Fuzzy Solution for Heat Equation Based on Generalized Hukuhara Differentiability. Fuzzy Sets and Systems, 265, 1-23. https://doi.org/10.1016/j.fss.2014.11.009

[10] Anastassiou, G.A. (2010) Fuzzy Mathematics: Approximation Theory. Springer, Berlin. https://doi.org/10.1007/978-3-642-11220-1

[11] Puri, M.L. and Ralescu, D.A. (1986) Fuzzy Random Variables. Journal of Mathematical Analysis and Applications, 114, 409-422.

https://doi.org/10.1016/0022-247X(86)90093-4

[12] Ralescu, D.A. (1999) Fuzzy Random Variables Revisited. In: Höhle, U. and Rodabaugh, S.E., Eds., Mathematics of Fuzzy Sets. The Handbook of Fuzzy Sets Series, Springer, Boston, 701-710. https://doi.org/10.1007/978-1-4615-5079-2_16

[13] Stefanini, L., Sorini, L. and Guerra, M.L. (2008) Fuzzy Numbers Fuzzy Arithmetic. John Wiley and Sons, Ltd., Hoboken.

[14] Bede, B. and Stefanini, L. (2013) Generalized Differentiability of Fuzzy-Valued Functions. Fuzzy Sets and Systems, 230, 119-141.

https://doi.org/10.1016/j.fss.2012.10.003

[15] Duminil-Copin, H. (2013) Mouvement Brownien et introduction au calcul stochastique. University of Geneva, Geneva.

[16] Aggoun, L. and Elliott, R.J. (2004) Measure Theory and Filtering Introduction and Application. Cambridge University Press, Cambridge.

https://doi.org/10.1017/CBO9780511755330

[17] Buckley, J.J. and Feuring, T. (2000) Fuzzy Differential Equations. Fuzzy Sets and Systems, 110, 43-54. https://doi.org/10.1016/S0165-0114(98)00141-9

[18] Bouleau, N. (1988) Processus Stochastiques et Applications. Hermann, Paris.

[19] Lamberton, D. and Lapeyre, B. (2012) Introduction au calcul stochastique appliqué à la finance. Ellipse, Minneapolis. 